\title{
Tribbles homolog 3 is induced by high glucose and associated with apoptosis in human endothelial cells
}

\author{
LING GUO $^{1,2}$, ZHONG-XIU GUO ${ }^{3}$, HUI-PING GONG ${ }^{4}$, \\ YUAN-YUAN SHANG ${ }^{1}$, MING ZHONG $^{1}$, YUN ZHANG ${ }^{1}$ and WEI ZHANG ${ }^{1}$
}

${ }^{1}$ Key Laboratory of Cardiovascular Remodelling and Function Research, Chinese Ministry of Education and Chinese Ministry of Health, Department of Cardiology, Qilu Hospital, Shandong University; ${ }^{2}$ Department of Nephrology,

Qilu Hospital of Shandong University, Jinan, Shandong 250012; ${ }^{3}$ Department of Cardiology,

The Central Hospital of Taian, Taian, Shandong 271000; ${ }^{4}$ Department of Cardiology,

The Second Hospital of Shandong University, Jinan, Shandong 250012, P.R. China

Received June 6, 2014; Accepted February 23, 2015

DOI: $10.3892 / \mathrm{mmr} .2015 .3576$

\begin{abstract}
Tribbles homolog 3 (TRIB3) is an intracellular kinase-like molecule that modifies cellular survival and metabolism. The present study aimed to investigate the function of TRIB3 regulation in the process of high glucose-induced apoptosis in endothelial cells, with the aim of identifying a novel intervention target for the prevention and treatment of diabetes mellitus. Human umbilical vein endothelial cells (HUVECs) grown in medium with various concentrations of glucose $(5.5,10,20,30$ and $40 \mathrm{mmol} / \mathrm{l})$ were assessed for mRNA expression of TRIB1, TRIB2 and TRIB3 using reverse transcription quantitative polymerase chain reaction. In addition, protein expression of TRIB3 was examined using western blot analysis. Immunofluorescence staining was performed in order to determine the distribution and localization of TRIB3 in HUVECs. Furthermore, cells grown in normal $(5.5 \mathrm{mmol} / \mathrm{l})$ or high glucose (HG; $30 \mathrm{mmol} / \mathrm{l})$ medium were subjected to TRIB3 inhibition through small interfering (si)RNA knockdown. These cells were then examined in order to determine whether TRIB3 upregulation was associated with endothelial cell apoptosis. HUVECs treated with 30 and $40 \mathrm{mmol} / \mathrm{l}$ glucose for $48 \mathrm{~h}$ and $72 \mathrm{~h}$ showed significantly lower survival rates compared with those treated with normal glucose levels. In addition, slight but not significant increases in TRIB1 and TRIB2 mRNA expression were observed in HUVECs incubated with various concentrations of glucose for different durations. By contrast, TRIB3 mRNA expression
\end{abstract}

Correspondence to: Professor Wei Zhang, Key Laboratory of Cardiovascular Remodelling and Function Research, Chinese Ministry of Education and Chinese Ministry of Health, Department of Cardiology, Qilu Hospital, Shandong University, 107 Wen Hua Xi Road, Jinan, Shandong 250012, P.R. China

E-mail: zhangwsdu@163.com

Key words: tribbles 3, umbilical endothelial cells, high glucose, atherosclerosis, apoptosis was increased 7.2-fold following incubation with HG. Western blot analysis revealed a 5.44-fold increase in TRIB3 protein levels in cells grown in HG medium for $24 \mathrm{~h}$ compared with those grown in normal medium. Immunostaining assays revealed a markedly higher and well-defined nucleolar fluorescence intensity for TRIB3 expression at $24 \mathrm{~h}$ in HG medium compared with that of the control group. Furthermore, the apoptotic rate of HG-treated TRIB3 siRNA-transfected HUVECs was significantly increased compared with that of those transfected with control siRNA In conclusion, the results of the present study suggested that TRIB3 was associated with high glucose-induced HUVECs apoptosis, which was attenuated following transfection with TRIB3 siRNA.

\section{Introduction}

Macroangiopathy is a major cause of mortality and morbidity in diabetes mellitus. In addition, atherosclerotic and/or arteriosclerotic changes in the cardiovascular system may result in the development and progression of cardiovascular diseases associated with diabetes (1). Endothelial dysfunction is recognized as one of the early and prominent stages in the formation and development of atherosclerotic lesions (2), in which endothelial proliferation and apoptosis have been observed (3). Impairment of endothelial function has been reported to occur in the early stage of diabetes, prior to clinically detectable angiopathy and hypertension (4). Hyperglycemia has been demonstrated to disrupt the cell cycle, increase DNA damage, delay cell replication and induce apoptosis in endothelial cells (5-8). The Akt (protein kinase B)/phosphatidylinositol 3-kinase (PI3K) pathway has a critical role in insulin signaling as well as cell apoptosis $(9,10)$. Molecular events linking high glucose with endothelial cell apoptosis were reported to be involved in the nuclear factor- $\mathrm{\kappa B}$-associated upregulation of cyclooxygenase- 2 as well as the increased production of reactive oxygen species via the Akt/PI3K pathway in human umbilical vein endothelial cells (HUVECs) $(9,11)$.

A previous study reported that tribbles homolog (TRIB) 3, a mammalian homolog of Drosophila tribbles, may be associated with modulated glucose metabolism through directly 
binding to Akt in the livers of diabetic mice (10). In addition, TRIB3 protein overexpression was reported to result in hyperglycemia and inhibit the activity of Akt (10). Subsequent clinical studies have proposed that functional TRIB3 missense polymorphisms may be associated with hyperinsulinemia, dyslipidemia and cardiovascular disease $(12,13)$. Another previous study demonstrated that the TRIB3 Q84R polymorphism increased the risk of metabolic syndrome and insulin resistance (14). In addition, the R84 allele was found to be associated with a predisposition to carotid atherosclerosis. These previous studies demonstrated a link between TRIB3 and atherosclerosis in diabetes; therefore, the present study aimed to investigate the involvement of TRIB3 protein in endothelial cell dysfunction. In order to investigate the effect of TRIB3 on endothelial cell dysfunction, the present study stimulated HUVECs with high glucose concentrations and transfected with an anti-TRIB3 inhibitor. The expression levels of miR-21 and HUVECs dysfunction were subsequently measured.

\section{Materials and methods}

Cell culture. HUVECs were purchased from the China Center for Type Culture Collection (Wuhan University, Wuhan, China). Cells were grown in endothelial cell basal medium containing M199 (Gibco-BRL, Carlsbad, CA, USA), 10\% fetal calf serum (Tianjin Lisheng Pharmaceutical Co., Ltd., Tianjin, China), $40 \mathrm{ng} / \mathrm{ml}$ growth factors (rHVEGF; cat. no. 676472; Chemicon, Billerica, MA USA), $100 \mathrm{U} / \mathrm{ml}$ penicillin and $100 \mu \mathrm{g} / \mathrm{ml}$ streptomycin (Beyotime Institute of Biotechnology, Shanghai, China) at $37^{\circ} \mathrm{C}$ with $5 \% \mathrm{CO}_{2}$ in a humidified incubator. Confluent HUVECs were used for experiments between passages 3 and 5 .

Assays for cell viability. A methyl thiazolyl tetrazolium (MTT) assay was performed in order to determine the cell viability of HUVECs at various concentrations of glucose. Cells were seeded at a density of $5 \times 10^{3}$ cells/well into 96 -well plates for $24 \mathrm{~h}$. Confluent cells were then incubated in serum-free cell culture medium with various concentrations of glucose (5.5, $10,20,30$ and $40 \mathrm{mmol} / \mathrm{l})$ for 24,48 or $72 \mathrm{~h}$ at $37^{\circ} \mathrm{C}$ with $5 \% \mathrm{CO}_{2}$. MTT solution (20 $\mu \mathrm{l} /$ well; Sigma-Aldrich, St. Louis, MO, USA) was then added and the samples were incubated for a further $4 \mathrm{~h}$ at $37^{\circ} \mathrm{C}$ with $5 \% \mathrm{CO}_{2}$. The absorbance of the samples was read at $490 \mathrm{~nm}$ (Model 1450; Bio-Rad Laboratories, Inc., Hercules, CA, USA).

Reverse transcription quantitative polymerase chain reaction (RT-qPCR). HUVECs were treated with various concentrations of glucose and for different durations as follows: $30 \mathrm{mmol} / 1$ glucose for $0,4,8,12,24,48$ or $72 \mathrm{~h}$; $5.5,10,20,30$ and $40 \mathrm{mmol} / 1$ glucose for $24 \mathrm{~h}$; and control (5.5 mmol/l), high $(30 \mathrm{mmol} / \mathrm{l})$ and hypertonic mannitol (25 mmol/l; Shijiazhuang Siyao Co., Ltd., China) concentrations for $0,4,7,12,24,48$ and $72 \mathrm{~h}$. Mannitol was used to exclue te effect of high glucose osmotic pressiure on the cells. Total RNA was extracted from HUVECs using TRIzol reagent (Invitrogen Life Technologies, Waltham, MA, USA). qPCR with cDNA was performed using a Real-Time Fluorescent SYBR Green I PCR kit (Takara Bio, Inc., Dalian, China). The cDNA primers (Shanghai Boya
Biotechnology Co., Ltd., Shanhai, China) used were as follows: TRIB1 sense, 5'-GCTGTGCATCCACACTGGAC-3' and antisense, 5'-GCGATGGCAGCTGGATGTAA-3'; TRIB2 sense, 5'-CTCAAGCTGCGGAAATTCATCTTTA-3' and antisense, 5'-TGGTGTTCAAGATCTCTGGGCTTAC-3'; TRIB3 sense, 5'-GTCTGGTCCTGCGTGATCTCAA-3' and antisense, 5'-GTATG AGGCCCGTGAGCTGAGT-3'; $\beta$-actin sense, 5'-TGGACATCCGCAAAGAC-3' and antisense, 5'-GAAAGGGTGTAACGCAACTA-3' (Shanghai Boya Biotechnology Co., Ltd., Shanghai, China). mRNA expression levels were calculated relative to those of $\beta$-actin.

Immunofluorescence staining and western blot analysis of TIRB 3 protein levels. In order to investigate effect of glucose on TRIB3 protein levels, immunofluorescence staining and western blot analysis were performed. For immunofluorescence staining, HUVECs were treated with high glucose (HG; $30 \mathrm{mmol} / \mathrm{l}$ ) for 24 or $48 \mathrm{~h}$ at $37^{\circ} \mathrm{C}$ with $5 \% \mathrm{CO}_{2}$. Cells were grown on glass coverslips and fixed with $4 \%$ paraformaldehyde (Tianjin Guangcheng Chemical Reagents Co., Ltd., Tianjin, China) for $30 \mathrm{~min}$ at room temperature, then permeabilized with a blocking solution containing $0.3 \%$ Triton X-100 and 5\% bovine serum albumin (both Tianjin Lisheng Pharmaceutical Co., Ltd.) in phosphate-buffered saline (PBS). Following incubation with rabbit polyclonal immunoglobulin (Ig)G TRIB3 primary antibodies (1:50; cat. no. NB100-56398; Imgenex, Littleton, CO, USA) overnight at $4^{\circ} \mathrm{C}$, fluorescein-isothiocyanate (FITC)-conjugated anti-rabbit secondary antibodies (1:100; cat. no. 35552; Beijing Zhongshan Golden Bridge Biotechnology Co., Ltd., Beijing China) were added and incubated for $1 \mathrm{~h}$ at room temperature. Nuclei were counterstained with DAPI (Nanjing KGI Biotechnology Development Co., Ltd, Nanjing, China) for $1 \mathrm{~min}$. Cells were then observed and fluorescence images were captured using an inverted fluorescence microscope (IX71-A12FL/PH; Olympus Corp., Tokyo, Japan).

For western blot analysis, HUVECs were exposed to HG (30 mmol/l) for 24,48 or $72 \mathrm{~h}$. TRIB3 protein concentrations were determined using a Bicinchoninic Acid Protein Assay Kit (Beyotime Institute of Biotechnology) according to the manufacturer's instructions. Protein samples were electrotransferred onto 20\% SDS-polyacrylamide gels and then immobilized to nitrocellulose membranes (Bio-Rad Laboratories, Inc.). The membranes were blocked and incubated with rabbit polylclonal IgG TRIB3 primary antibodies (1:800; cat. no. NB100-56398; Imgenex) overnight at $4^{\circ} \mathrm{C}$. Following washing three times with $1 \mathrm{X}$ Tris-buffered saline containing tween-20 for 5 mins, the membranes were incubated with goat anti-rabbit IgG secondary antibodies conjugated to horseradish peroxidase (1:100; cat. no. ZF-0315; Pierce Biotechnology, Inc., Rockford, IL, USA). The immunoreactive bands were quantified using a FluorChem 9900-50 gel documentation imaging system (Alpha Innotech, San Leandro, CA, USA).

Small interfering (si)RNA and transfection. siRNA against humanTIRB3(sense,5'-CGAGCUCGAAGUGGGCCCCTT-3' and antisense, 5'-GGGGCCCACUUCGAGCUCGTT-3') were designed and synthesized by Zimmer Medical International Trading (Shanghai, China). A nonspecific siRNA duplex 


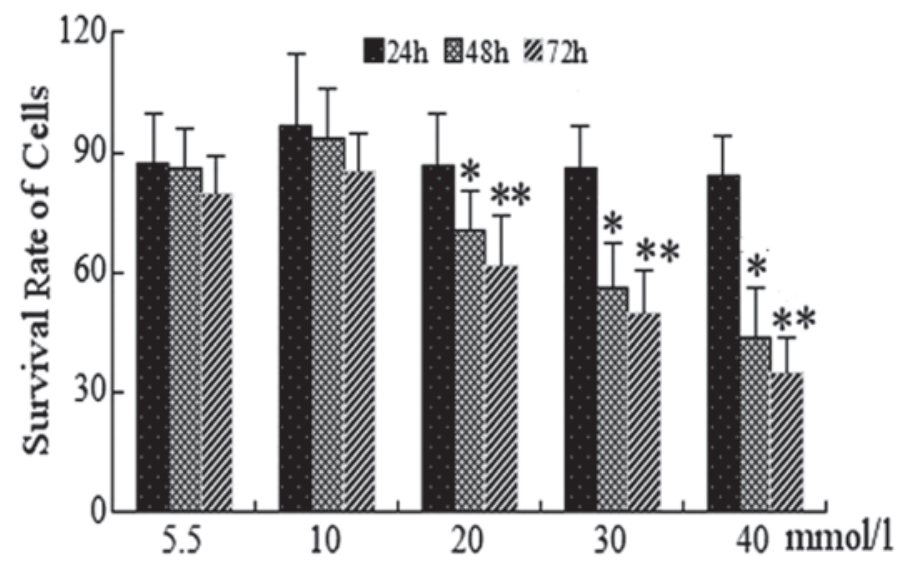

Figure 1. Survival rate of HUVECs incubated with various glucose concentrations for different durations. An MTT assay was used to determined the viability of HUVECs following incubation with glucose at various concentrations (5.5, 10, 20, 30 and $40 \mathrm{mmol} / \mathrm{l})$ for 24, 48 and $72 \mathrm{~h}$. Values are presented as the mean \pm standard deviation. ${ }^{*} \mathrm{P}<0.05,{ }^{* * *} \mathrm{P}<0.01$ vs. $24 \mathrm{~h}$. HUVECs, human umbilical vein endothelial cells.

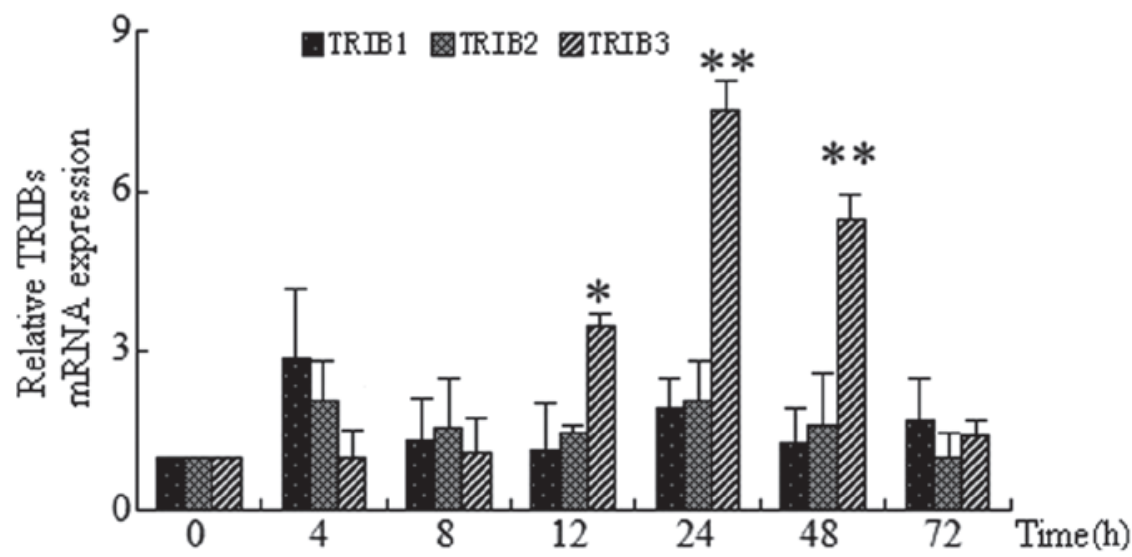

Figure 2. Time-dependent expression of TRIB mRNA in HUVECs incubated with high glucose. HUVECs were incubated with $30 \mathrm{mmol} / \mathrm{l}$ glucose for $0,4,7,12,24,48$ and $72 \mathrm{~h}$. Reverse transcription quantitative polymerase chain reaction was then used to analyze the mRNA expression of TRIB1, TRIB2 and TRIB3. Values were calculated relative to the expression of $\beta$-actin and are presented as the mean \pm standard deviation. "P<0.05 and ${ }^{* *} \mathrm{P}<0.01$, as compared with at $0 \mathrm{~h}$. TRIB, tribbles homolog gene; HUVECs, human umbilical vein endothelial cells.

(sense, 5'-UUCUCCGAACGUGUCACGUTT-3' and antisense, 5'-ACGUGACACGUUCGGAGAATT-3') (Zimmer Medical International Trading) was used as a control oligonucleotide. HUVECs were grown to $50 \%$ confluence in 12 -well plates and Opti-Minimal Essential Medium (Thermo Fisher Scientific, Waltham, MA, USA). Cells were transfected with 400 pmol siRNA molecules (at a concentration of $150 \mathrm{pmol} / 10 \mathrm{~cm}^{2}$ ) using Lipofectamine 2000 (Invitrogen Life Technologies). Following $6 \mathrm{~h}$ of incubation at $37^{\circ} \mathrm{C}$ with $5 \% \mathrm{CO}_{2}$, the medium was replaced with complete cell culture medium or $\mathrm{HG}(30 \mathrm{mmol} / \mathrm{l})$ medium for $48 \mathrm{~h}$ at $37^{\circ} \mathrm{C}$ with $5 \% \mathrm{CO}_{2}$.

FITC-Annexin $V$ and propidium iodide (PI) double staining. Cells $\left(0.5 \times 10^{6} /\right.$ well $)$ were seeded into 6-well plates, which were treated with or without TRIB3 or control siRNA in the presence of normal glucose $(5.5 \mathrm{mmol} / \mathrm{l})$ or $\mathrm{HG}(30 \mathrm{mmol} / \mathrm{l})$, followed by incubation for $48 \mathrm{~h}$ at $37^{\circ} \mathrm{C}$ with $5 \% \mathrm{CO}_{2}$. Following $0.125 \%$ trypsin-EDTA (Gibco Life Technologies, Carlsbad, CA, USA) digestion, the cell pellet was washed twice with ice-cold PBS. Subsequently, $400 \mu \mathrm{l}$ binding buffer (Sigma-Aldrich) and $5 \mu \mathrm{l}$ FITC-labeled Annexin V (Bipec Biopharma Corporation,
Cambridge, MA, USA) were added and cells were then incubated in the dark at $4-8^{\circ} \mathrm{C}$ for $15 \mathrm{~min}$. The PI solution $(10 \mu \mathrm{l}$; Bipec Biopharma Corporation, Cambridge, MA, USA) was added and cells were incubated in the dark for a further $5 \mathrm{~min}$. A FACScan flow cytometer (Becton Dickinson, Franklin Lakes, NJ, USA) was then used to determine the apoptotic rate of cells. The ratio of PI-positive to Annexin V-positive cells was used as a negative control.

Statistical analysis. Values are presented as mean \pm standard deviation. An unpaired t-test and one-way analysis of variance followed by the least significant difference post hoc test were used to evaluate the data. Statistical analyses were performed using SPSS 15.0 software (SPSS, Inc., Chicago, IL, USA). $\mathrm{P}<0.05$ was considered to indicate a statistically significant difference between values.

\section{Results}

Effect of various glucose concentrations on HUVEC survival rates. An MTT assay was used to determine the survival rate 


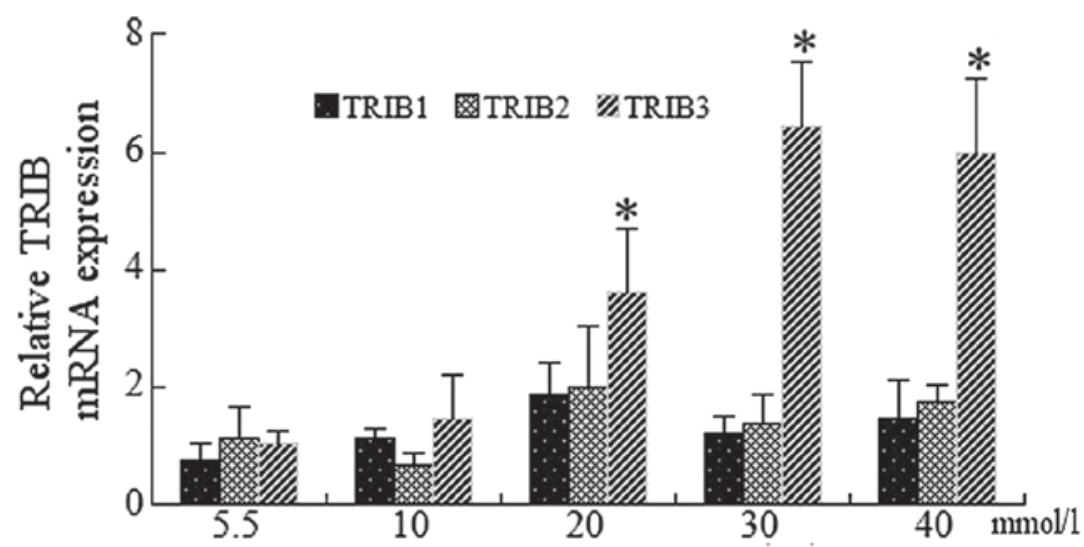

Figure 3. Dose-dependent effect of glucose on TRIB mRNA expression in HUVECs. HUVECs were incubated with various concentrations of glucose $(5.5,10,20,30$ and $40 \mathrm{mmol} / \mathrm{l})$ for $24 \mathrm{~h}$. Reverse transcription quantitative polymerase chain reaction was then used to analyze the mRNA expression of TRIB1, TRIB2 and TRIB3. Values were calculated relative to the expression of $\beta$-actin and are presented as the mean \pm standard deviation. ${ }^{*} \mathrm{P}<0.05$ vs. $5.5 \mathrm{mmol} / \mathrm{l}$. TRIB, tribbles homolog gene; HUVECs, human umbilical vein endothelial cells.

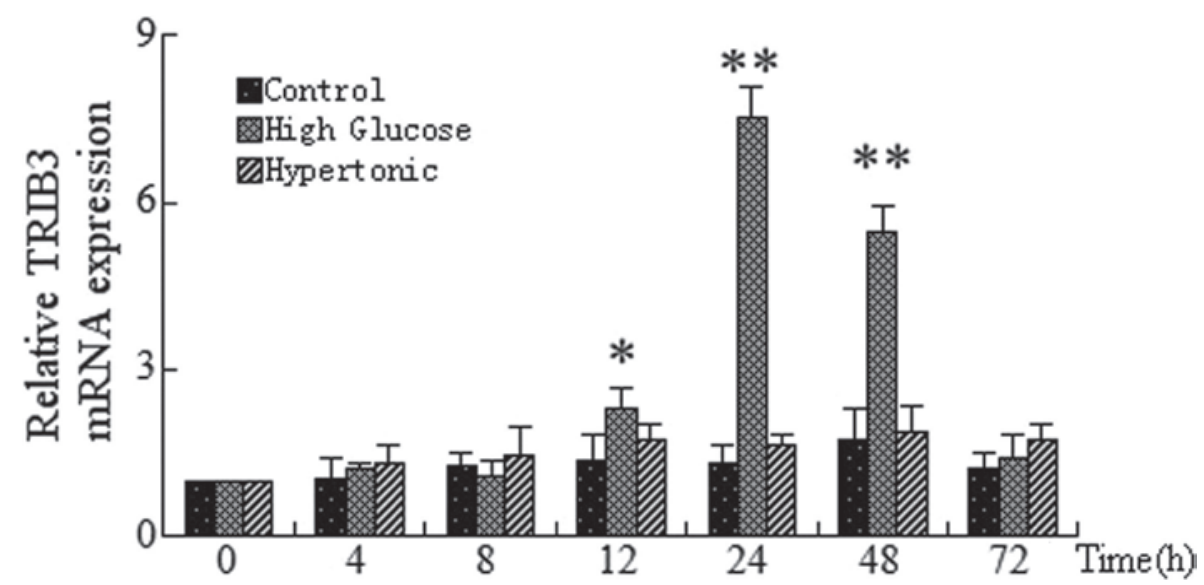

Figure 4. Time-dependent expression of TRIB3 mRNA in HUVECs incubated under control, high glucose and hypertonic conditions. HUVECs were incubated with control $(5.5 \mathrm{mmol} / \mathrm{l})$, high $(30 \mathrm{mmol} / \mathrm{l})$ and hypertonic mannitol concentrations for $0,4,7,12,24,48$ and $72 \mathrm{~h}$. Reverse transcription polymerase chain reaction was then used to analyzed the mRNA expression of TRIB3. Values were calculated relative to the expression of $\beta$-actin and are presented as the mean \pm standard deviation. ${ }^{*} \mathrm{P}<0.05$ and ${ }^{* * *} \mathrm{P}<0.01$, as compared with at $0 \mathrm{~h}$. TRIB3, tribbles homolog 3 gene; HUVECs, human umbilical vein endothelial cells; Control, normal glucose.

of cells following treatment with various glucose concentrations $(5.5,10,20,30$ and $40 \mathrm{mmol} / \mathrm{l})$ for 24,48 and $72 \mathrm{~h}$. As shown in Fig. 1, at $24 \mathrm{~h}$ no significant differences were identified in the cell viability of HUVECs treated with 30 or $40 \mathrm{mmol} / 1$ glucose compared with that of the normal glucose control group ( $5.5 \mathrm{mmol} / \mathrm{l} ; \mathrm{P}>0.05)$. Under identical conditions, HUVECs treated with 30 and $40 \mathrm{mmol} / \mathrm{l}$ for 48 and $72 \mathrm{~h}$ demonstrated significantly reduced survival rates compared with that of the control group $(\mathrm{P}<0.05)$. No significant differences were observed in cell viability at glucose concentrations of 10 and $20 \mathrm{mmol} / \mathrm{l}$ at 24,48 or $72 \mathrm{~h}$ compared with that of the control group. Overall, the survival rates of HUVECs were found to be decreased in a concentration- and time-dependent manner following treatment with glucose (Fig. 1). Of note, the cell survival rate of HUVECs was $<50 \%$ at $40 \mathrm{mmol} / \mathrm{l}$ glucose; therefore, the $\mathrm{HG}$ groups were treated with $30 \mathrm{mmol} / \mathrm{l}$ glucose for subsequent experiments.

Effects of HG on TRIB mRNA expression. HUVECs were treated with $30 \mathrm{mmol} / 1$ glucose for $4,8,12,24,48$ or $72 \mathrm{~h}$. RT-qPCR was then used to determine the mRNA expression of TRIB1, TRIB2 and TRIB3. The results indicated that mRNAs of each TRIB gene were present in endothelial cells. In addition, TRIB3 gene expression was found to increase in a time-dependent manner in response to HG. TRIB3 expression peaked at $24 \mathrm{~h}$ (7.2-fold vs. control; $\mathrm{P}<0.01$ ), which then decreased by $48 \mathrm{~h}$; at $72 \mathrm{~h}$ TRIB3 expression was not significantly increased. By contrast, under identical conditions no significant increases were observed for TRIB1 and TRIB2 mRNA expression in HUVECs at any time-point (Fig. 2).

Effect of various glucose concentrations on TRIB $m R N A$. mRNA expression of the TRIB genes was examined in HUVECs incubated with 5.5, 10, 20, 30 and $40 \mathrm{mmol} / \mathrm{l}$ glucose for $24 \mathrm{~h}$. RT-qPCR analysis of endothelial cells revealed significant increases in TRIB3 expression at 30 and $40 \mathrm{mmol} / \mathrm{l}$ glucose compared with that of the control $(\mathrm{P}<0.01)$; of note, TRIB3 expression was highest following treatment with $30 \mathrm{mmol} / \mathrm{l}$ glucose (Fig. 3). Although mRNA expression of TRIB1 and TRIB2 showed slight increases, the values did not reach statistical significance (data not shown). 


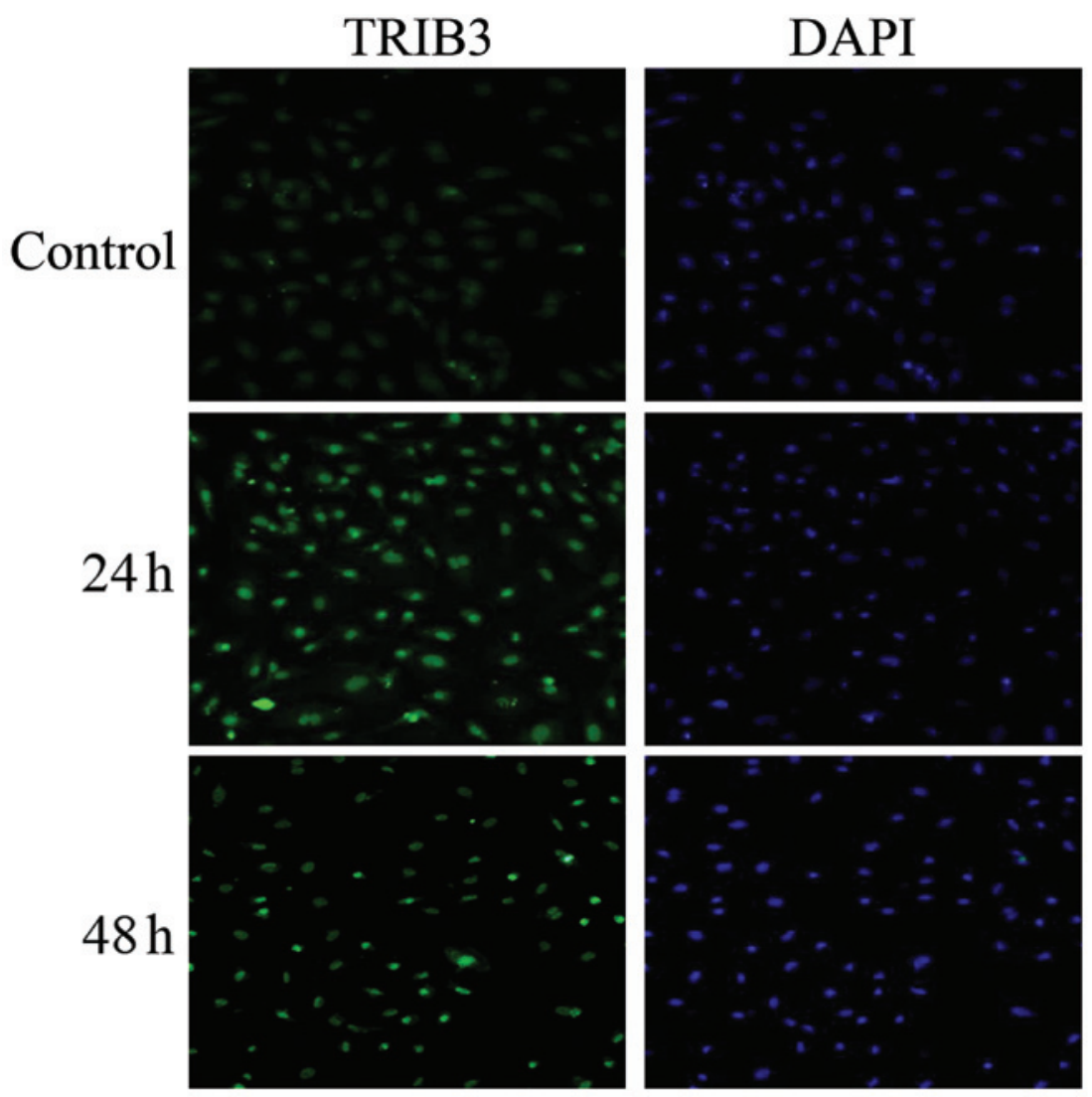

Figure 5. Subcellular localization of TRIB3 was visualized in high glucose-treated HUVECs. Immunofluorescence staining was used to observe TRIB3 expression in HUVECs incubated with high glucose $(30 \mathrm{mmol} / \mathrm{l})$ for $24 \mathrm{~h}$ and $48 \mathrm{~h}$. TRIB3, tribbles homolog 3 gene; HUVECs, human umbilical vein endothelial cells.
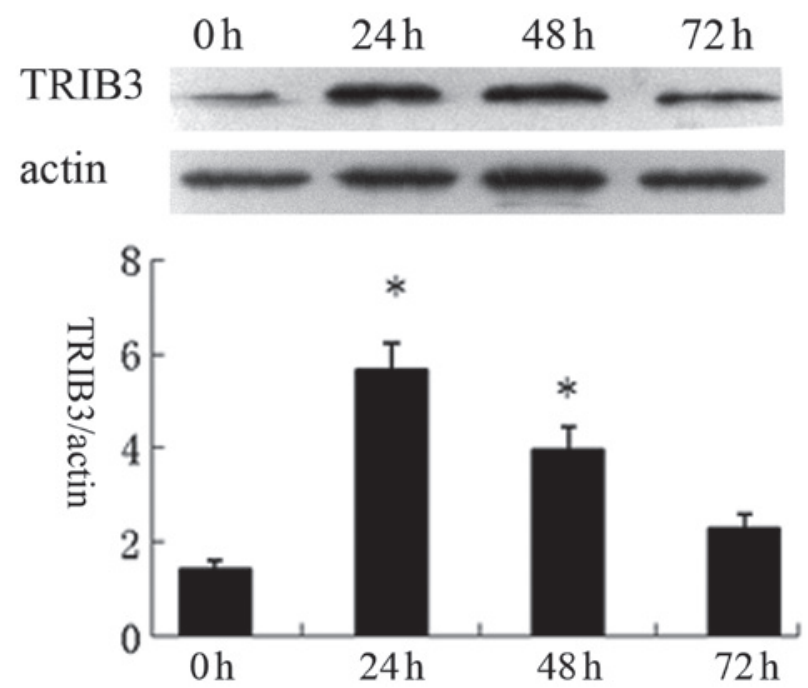

Figure 6. Time-dependent protein expression of TRIB3 in HUVECs incubated with high glucose. HUVECs were incubated with $30 \mathrm{mmol} / 1$ glucose for $0,24,48$ and $72 \mathrm{~h}$. Western blot analysis was then used to determine the protein expression of TRIB3. Values were calculated relative to the expression of actin and are presented as the mean \pm standard deviation of arbitrary densitometric units. " $\mathrm{P}<0.05$, as compared with at $0 \mathrm{~h}$. TRIB3, tribbles homolog 3 gene; HUVECs, human umbilical vein endothelial cells.

Effect of hypertonic glucose on mRNA expression of TRIB3. In order to determine whether high glucose is responsible for high glucose-induced TRIB3 expression, TRIB3 mRNA expression was examined in HUVECs treated with HG, hypertonia and normal glucose conditions for 4, 8, 12, 24, 48 and 72 h. RT-qPCR analysis revealed that TRIB3
mRNA expression was significantly increased following 12, 24 and $48 \mathrm{~h}$ of $\mathrm{HG}$ treatment compared with that of the normal glucose group at each time-point, with the highest TRIB3 expression observed at $24 \mathrm{~h}$ post treatment (Fig. 4). By contrast, cells grown in hypertonic glucose showed no significant differences in TRIB3 mRNA expression levels 

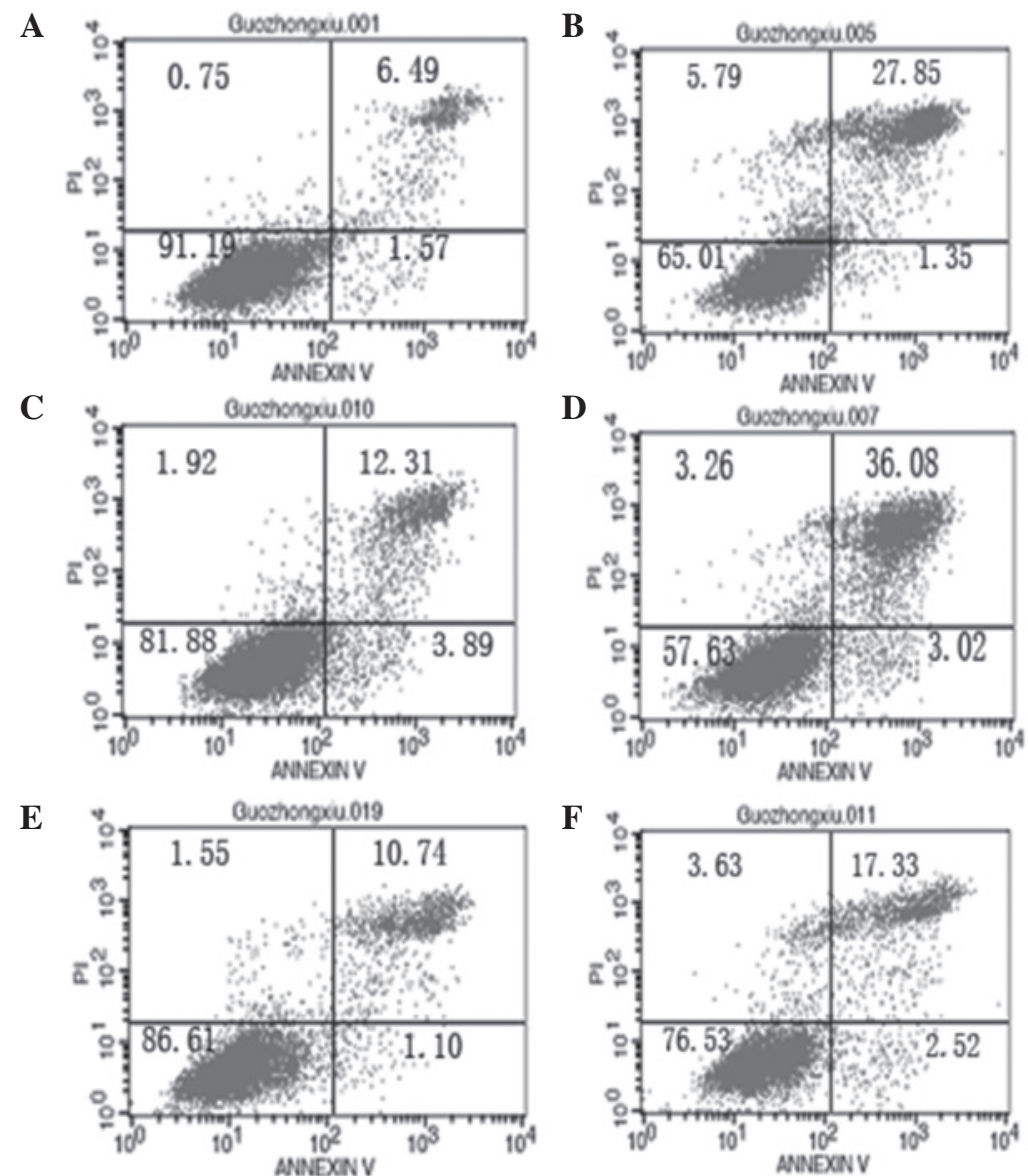

Figure 7. Assessment of HUVECs apoptosis using flow cytometric analysis with Annexin V and PI staining. Cells were treated with and without TRIB3 siRNA or control siRNA in the presence of normal glucose $(5.5 \mathrm{mmol} / \mathrm{l})$ or $\mathrm{HG}(30 \mathrm{mmol} / \mathrm{l})$ medium for $48 \mathrm{~h}$ as follows: (A) Normal glucose; (B) HG; (C) normal glucose + control siRNA; (D) HG + control siRNA; (E) normal glucose + TRIB3 siRNA; and (F) HG + TRIB3 siRNA. HUVECs, human umbilical vein endothelial cells; PI, propidium iodide; TRIB3, tribble homolog 3; siRNA, small interfering RNA; HG, high glucose.

compared with those of the normal glucose group at each time-point (Fig. 4).

Effects of HG on subcellular localization and protein expression of TRIB3. The distribution and localization of TRIB3 in the endothelial cells was assessed using immunofluorescence staining. As shown in Fig. 5, the immunostaining intensity of TRIB3 showed increased expression in the nuclei of cells cultured in HG medium compared with that of cells treated with normal glucose. However, TRIB3 expression was not observed in the cytoplasm of HUVECs. Furthermore, these immunostaining assays confirmed that expression of TRIB3, as measured by nucleolar fluorescence and immunofluorescence staining, was strongest at $24 \mathrm{~h}$, followed by exposure for $48 \mathrm{~h}$. Western blot analysis revealed that HUVECs grown in HG medium for $24 \mathrm{~h}$ exhibited a 5.44-fold increase in TRIB3 protein levels, which then decreased, although expression was still significant compared with the control group, following exposure for $48 \mathrm{~h}$. However, exposure of the cells to HG for $72 \mathrm{~h}$ had no significant effect on TRIB3 expression compared with that of the control (Fig. 6). These results were consistent with the results of the immunofluorescence experiment.

Effect of TRIB3 siRNA on HUVEC apoptosis following HG treatment. As shown in Fig. 7, the effect of TRIB3 siRNA on HUVEC apoptosis was examined under high glucose conditions for $48 \mathrm{~h}$. In the present study, siRNA exhibited an efficient inhibitory effect on TRIB3 expression and cell apoptosis at a concentration of $150 \mathrm{pmol} / 10 \mathrm{~cm}^{2}$. TRIB3 gene expression was reduced by $90 \%$ at $48 \mathrm{~h}$ post-transfection. In addition, cell apoptosis was reduced by $42 \%$ in the transfected cells grown in HG medium for $48 \mathrm{~h}$, as compared with the cells grown in normal medium. The apoptotic fraction of cells grown in HG medium was significantly reduced, as compared with the cells grown in normal glucose medium $(\mathrm{P}<0.05)$. In addition, TRIB3 siRNA-transfected HUVECs cultured in HG medium had a significantly higher rate of cell apoptosis compared with that of HG-treated cells transfected with control siRNA $(\mathrm{P}<0.05)$ (Fig. 7).

\section{Discussion}

The aim of present study was to obtain insights into the role of human TRIB genes, particularly TRIB3, in endothelial cell dysfunction in vitro and provide evidence for the role of these genes in diabetes-associated atherosclerosis. In the current study, it was demonstrated that the expression of TRIB1, TRIB2 and TRIB3 were detected in endothelial cells following culture in high glucose medium. In addition, the results showed that genetic ablation of endogenous TRIB3 by siRNA 
reduced endothelial cell apoptosis and promoted cell survival. Therefore, the present study demonstrated that increased TRIB3 expression may regulate HG- or diabetes-induced endothelial cell apoptosis.

In one study of a cell model, high ambient glucose concentrations were shown to modulate the mRNA expression of fibronectin, collagen, tissue-type plasminogen activator and plasminogen activator inhibitor; in addition, high glucose was demonstrated to induce delayed replication and excess cell death in cultured vascular endothelial cells (15). Endothelial dysfunction has been hypothesized to have an important role in the progression and pathogenesis of vascular complications in diabetes. Numerous studies have reported that the apoptosis of endothelial cells was prominent in models of hyperglycemia in vitro $(16,17)$. In the present study, high glucose concentrations were found to induce apoptosis in HUVECs, which was in line with previous findings (15-17). Furthermore, HUVEC survival rates were found to decrease in a concentration- and time-dependent manner. However, the specific mechanisms of high glucose-induced apoptotic endothelial cell death remains to be fully elucidated.

The human homolog of Drosophila tribbles (TRIB) was first identified using a genome-wide functional screen for components of inflammatory signaling networks (18). Drosophila tribbles was reported to be involved in the coordination of entry into mitosis as well as morphogenesis and cell fate determination during early embryogenesis (19). Overexpression of tribbles was reported to slow the cell cycle, while loss of tribbles function was associated with increased proliferation (20). In humans there are three mammalian tribbles-like proteins, TRIB1, TRIB2 and TRIB3 (21). TRIBs appear to function in a cell-type- and stimulus-specific manner. In response to inflammatory stimuli, it was demonstrated that TRIB1 was rapidly and transiently upregulated in aortic smooth muscle cells and monocytes, whilst a profound but delayed activation was observed in synoviocytes (22). In addition, TRIB2 expression was reported to be upregulated at $6 \mathrm{~h}$ post inflammatory stimuli (interleukin-1) in monocytes, whereas TRIB2 expression was significantly decreased in response to identical stimuli in synovial fibroblasts (22). Furthermore, TRIB3 expression was differentially regulated in the various cell types examined; low TRIB3 mRNA levels were detected at 3 and $6 \mathrm{~h}$ following inflammatory stimuli in synovial fibroblasts and vascular smooth muscle cells; by contrast, in THP-1 cells, TRIB3 expression was significantly upregulated, with the highest levels observed at $10 \mathrm{~h}$ following stimuli. However, it remained to be elucidated whether the TRIB genes were involved in the regulation of endothelial cell apoptosis in response to high glucose or diabetes. The results of the present study indicated that increasing concentrations of glucose were able to stimulate TRIB1, TRIB2 and TRIB3 mRNA expression in HUVECs. Of note, TRIB1 and TRIB2 genes were transiently, but not significantly, upregulated in response to high glucose levels compared with that of the normal glucose group. However, TRIB3 expression was significantly upregulated in HUVECs in response to high glucose concentrations, with the highest levels observed following $24 \mathrm{~h}$ of culture or at $30 \mathrm{mmol} / \mathrm{l}$ glucose. The pattern of TRIB3 regulation was observed to be time- and concentration-dependent. Furthermore, immunofluorescence staining following
HG treatment revealed that TRIB3 protein was predominantly localized in the nuclei of endothelial cells. Comparable results regarding the subcellular localization of TRIB3 were reported in a study by Ord et al (23). This previous study demonstrated that the TRIB3-green fluorescent protein fusion protein resided primarily in the nuclei of transfected cells, including cos-7, GT1-7, CHO, HeLa and HEK293 cells (23).

A previous study demonstrated that TRIB3 was upregulated in response to fasting and diabetes; therefore, it was proposed that TRIB3 may have a major role in hepatic insulin resistance (10). Analyses of TRIB3 expression demonstrated that TRIB3 levels were highest in liver tissues; however, TRIB3 was also detected in the heart, kidney, lung, skin, small intestine and stomach, although it was not found to be located in skeletal muscle (24). Altered TRIB3 expression induced by various stimuli was reported to be highly cell- and/or species-type specific. In PC-3 prostate cancer cells, a glucose or amino acid deficiency resulted in a substantial increase in TRIB3 protein levels and this increase was reversed following the addition of fresh nutrients (25). In addition, hypoxia, osmotic stress or serum starvation did not exert a significant effect on TRIB3 expression (25). In another study, TRIB3 mRNA was found to be elevated in 3T3-L1 adipocytes and L6 myotubes exposed to low glucose or glucose-free medium and in 3T3-L1 adipocytes exposed to dexamethasone (26). However, in the present study, the results of the in vitro analysis of TRIB3 expression in HUVECs following glucose stimulation were not consistent with these previous findings. TRIB3 expression was analyzed using RT-qPCR, western blot analysis and immunofluorescence staining, the results of which revealed that TRIB3 expression was highest in cells incubated in HG medium for $24 \mathrm{~h}$, followed by that of exposure for $48 \mathrm{~h}$. Furthermore, the present study reported that the increased expression of TRIB3 in response to high glucose was not mediated by glucose-associated hypertonia. This therefore indicated that rapid, high glucose and endoplasmic stress may induce the changes in the expression of TRIB3 in different histological types, including liver, adiopose tissue, heart, kidney, lung, skin, small intestine and stomach (24).

A previous study suggested that TRIB3 has important roles in the coordination of entry into mitosis as well as morphogenesis and cell fate determination through regulating the degradation of the CDC25 mitotic activator String (19). TRIB3 has been reported to be involved in cell death during endoplasmic reticulum stress due to the downregulation of its own induction through the repression of CCAAT/enhancer binding protein homologous protein/activating transcription factor 4 function $(27,28)$. Therefore, in the present study, a loss of function experiment was performed using TRIB3 siRNA knockdown to evaluate the effects of TRIB3 on the regulation of HUVEC apoptosis. siRNA is used to regulate gene expression through entering a multimeric nuclease complex that identifies target mRNA (29). In the present study, siRNA efficiently exhibited a pronounced inhibitory effect on TRIB3 expression and cell apoptosis at a concentration of $150 \mathrm{pmol} / 10 \mathrm{~cm}^{2}$. TRIB3 gene expression was reduced by $90 \%$ at $48 \mathrm{~h}$ following transfection. In addition, cell apoptosis was reduced by $42 \%$ in transfected cells grown in HG medium for $48 \mathrm{~h}$ compared with those grown in normal medium. Therefore the silencing of the TRIB3 gene by siRNA revealed that endogenous levels 
of TRIB3 protected HUVECs cells from apoptosis in response to high glucose.

In conclusion, the present study identified that TRIB1, TRIB2 and TRIB3 were present in HUVECs cells. In addition, these findings suggested that TRIB3 was associated with HG-induced HUVECs apoptosis and may partially mediate the formation and/or pathogenesis of atherosclerosis under diabetic conditions. Therefore, TRIB3 may be a potential therapeutic target for attenuating the progression of atherosclerosis in diabetes.

\section{Acknowledgements}

The present study was supported by research grants from the Independent Innovation Foundation of Shandong University (no. 2012JC034), the Research Award Fund for Outstanding Middle-aged and Young Scientist of Shandong Province (no. BS2010YY029), the Natural Science Foundation of Shandong Province (nos. ZR2009CM022, ZR2009CM025 and BS2009YY026), the National Natural Science Foundation of China (nos. 30971215, 81070141, 81100605, 81270352 and 81270287) and the National Basic Research Program of China (973 Program; no. 2013CB530700).

\section{References}

1. O'Neill MS, Veves A, Zanobetti A, et al Diabetes enhances vulnerability to particulate air pollution-associated impairment in vascular reactivity and endothelial function. Circulation 111: 2913-2920, 2005.

2. Davignon J and Ganz P: Role of endothelial dysfunction in atherosclerosis. Circulation 109 (21 Suppl 1): III27-III32, 2004.

3. Norata GD, Tonti L, Roma P and Catapano AL: Apoptosis and proliferation of endothelial cells in early atherosclerotic lesions: possible role of oxidised LDL. Nutr Metab Cardiovasc Dis 12: 297-305, 2002.

4. Guangda X and Yuhua W: Apolipoprotein e4 allele and endothelium-dependent arterial dilation in Type 2 diabetes mellitus without angiopathy. Diabetologia 46: 514-519, 2003.

5. Lorenzi M, Cagliero E and Toledo S: Glucose toxicity for human endothelial cells in culture. Delayed replication, disturbed cell cycle, and accelerated death. Diabetes 34: 621-627, 1985.

6. Lorenzi M, Montisano DF, Toledo S and Barrievx A: High glucose induces DNA damage in cultured human endothelial cells. J Clin Invest 77: 322-325, 1986.

7. Lorenzi M, Nordberg JA and Toledo S: High glucose prolongs cell-cycle traversal of cultured human endothelial cells. Diabetes 36: 1261-1267, 1987.

8. Kageyama S, Yokoo H, Tomita K, et al: High glucose-induced apoptosis in human coronary artery endothelial cells involves up-regulation of death receptors. Cardiovasc Diabetol 10: 73, 2011.

9. Sheu ML, Ho FM, Yang RS, et al: High glucose induces human endothelial cell apoptosis through a phosphoinositide 3-kinase-regulated cyclooxygenase-2 pathway. Arterioscler Thromb Vasc Biol 25: 539-545, 2005.

10. Du K, Herzig S, Kulkarni RN and Montminy M: TRIB3: a tribbles homolog that inhibits Akt/PKB activation by insulin in liver. Science 300: 1574-1577, 2003.

11. Radovits T, Lin LN, Zotkina J, et al: Poly(ADP-ribose) polymerase inhibition improves endothelial dysfunction induced by reactive oxidant hydrogen peroxide in vitro. Eur J Pharmacol 564: 158-166, 2007.
12. Prudente S, Hribal ML, Flex E, et al: The functional Q84R polymorphism of mammalian Tribbles homolog TRIB3 is associated with insulin resistance and related cardiovascular risk in Caucasians from Italy. Diabetes 54: 2807-2811, 2005.

13. Qi L, Heredia JE, Almrejos JY, et al: TRB3 links the E3 ubiquitin ligase COP 1 to lipid metabolism. Science 312: 1763-1766, 2006.

14. Gong HP, Wang ZH, Jiang H, et al: TRIB3 functional Q84R polymorphism is a risk factor for metabolic syndrome and carotid atherosclerosis. Diabetes Care 32: 1311-1313, 2009.

15. Baumgartner-Parzer SM, Wagner L, Pettermann M, et al: High-glucose-triggered apoptosis in cultured endothelial cells. Diabetes 44: 1323-1327, 1995.

16. Nakagami $\mathrm{H}$, Morishita $\mathrm{R}$, Yamamoto $\mathrm{K}$, et al: Phosphorylation of p38 mitogen-activated protein kinase downstream of bax-caspase-3 pathway leads to cell death induced by high D-glucose in human endothelial cells. Diabetes 50: 1472-1481, 2001.

17. Zou MH, Shi C and Cohen RA: High glucose via peroxynitrite causes tyrosine nitration and inactivation of prostacyclin synthase that is associated with thromboxane/prostaglandin $\mathrm{H}(2)$ receptor-mediated apoptosis and adhesion molecule expression in cultured human aortic endothelial cells. Diabetes 51: 198-203, 2002.

18. Kiss-Toth E, Wyllie DH, Holland K, et al: Functional mapping of Toll/interleukin-1 signalling networks by expression cloning. Biochem Soc Trans 33 (Pt 6): 1405-1406, 2005.

19. Mata J, Curado S, Ephrussi A and Rørth P: Tribbles coordinates mitosis and morphogenesis in Drosophila by regulating string/CDC25 proteolysis. Cell 101: 511-522, 2000.

20. Grosshans J and Wieschaus EA: A genetic link between morphogenesis and cell division during formation of the ventral furrow in Drosophila. Cell 101: 523-531, 2000.

21. Hegedus Z, Czibula A and Kiss-Toth E: Tribbles: a family of kinase-like proteins with potent signalling regulatory function. Cell Signal 19: 238-250, 2007.

22. Sung HY, Francis SE, Crossman DC and Kiss-Toth E: Regulation of expression and signalling modulator function of mammalian tribbles is cell-type specific. Immunol Lett 104: 171-177, 2006.

23. Ord D and Ord T: Mouse NIPK interacts with ATF4 and affects its transcriptional activity. Exp Cell Res 286: 308-320, 2003.

24. Okamoto H, Latres E, Liu R, et al: Genetic deletion of Trb3, the mammalian Drosophila tribbles homolog, displays normal hepatic insulin signaling and glucose homeostasis. Diabetes 56: 1350-1356, 2007.

25. Schwarzer R, Dames S, Tondera D, et al: TRB3 is a PI 3-kinase dependent indicator for nutrient starvation. Cell Signal 18: 899-909, 2006.

26. Yacoub Wasef SZ, Robinson KA, Berkaw MN and Buse MG: Glucose, dexamethasone and the unfolded protein response regulate TRB3 mRNA expression in 3T3-L1 adipocytes and L6 myotubes. Am J Physiol Endocrinol Metab 291: E1274-E1280, 2006.

27. Ord D, Meerits K and Ord T: TRB3 protects cells against the growth inhibitory and cytotoxic effect of ATF4. Exp Cell Res 313: 3556-3567, 2007.

28. Ohoka N, Hattori T, Kitagawa M, et al: Critical and functional regulation of CHOP (C/EBP homologous protein) through the N-terminal portion. J Biol Chem 282: 35687-35694, 2007.

29. Agrawal N, Dasaradhi PV, Mohmmed A, et al: RNA interference: biology, mechanism, and applications. Microbiol Mol Biol Rev 67: 657-685, 2003. 